\title{
Rotating vector solving method applied for nonlinear oscillator
}

\author{
L. Cveticanin ${ }^{1,2, a}$, P. Suchy ${ }^{3}$, I. Biro ${ }^{3}$, and M. Zukovic ${ }^{2}$ \\ 1 Doctoral School of Safety and Security Sciences, Obuda University, Budapest, Hungary \\ 2 Faculty of Technical Sciences, University of Novi Sad, Novi Sad, Serbia \\ ${ }^{3}$ Faculty of Engineering, University of Szeged, Szeged, Hungary
}

Received 14 January 2021 / Accepted 7 July 2021 / Published online 2 August 2021 (C) The Author(s) 2021

\begin{abstract}
Significant number of procedures for solving of the finite degree-of-freedom forced nonlinear oscillator are developed. For all of them it is common that they are based on the exact solution of the corresponding linear oscillator. For technical reasons, the aim of this paper is to develop a simpler solving procedure. The rotating vector method, developed for the linear oscillator, is adopted for solving of the nonlinear finite degree-of-freedom oscillator. The solution is assumed in the form of trigonometric functions. Assuming that the nonlinearity is small all terms of the series expansion of the function higher than the first are omitted. The rotating vectors for each mass are presented in the complex plane. In the paper, the suggested rotating vector procedure is applied for solving of a three-degree-of-freedom periodically excited oscillator. The influence of the nonlinear stiffness of the flexible elastic beam, excited with a periodical force, on the resonant properties of the system in whole is investigated. It is obtained that the influence of nonlinearity on the amplitude and phase of vibration is more significant for smaller values of the excitation frequency than for higher ones.
\end{abstract}

\section{Introduction}

The problem of the many degree-of-freedom (dof) linear oscillators with an external excitation is known for a long time. It is considered in almost all textbooks in theory of oscillation (see for example $[1,2]$ ). A system of $n$ masses is connected with linear elastic springs and dashpots with linear damping properties. One mass is harmonically excited. Motion of the system is modeled with coupled second-order differential equations with constant coefficients and the solution is obtained in analytical form. It is found that for the resonance case the particular solutions of the system are harmonic functions with frequency which corresponds to the excitation one, while the amplitude and phase angle of masses differ.

However, the most of oscillators are nonlinear. Then, the mathematical model of many degree-of-freedom oscillator is a system of coupled second-order nonlinear differential equations. To obtain the analytic solution for these equations is not an easy task. Various solving methods for the two mass systems with two degrees of freedom [3-9] are developed (see [10]): the energy balance method [11], the He's frequency-amplitude procedure [12], the He's variation approach [13], the higher order Hamiltonian approach [14] etc. For all of them it is common that the result is unable to give an exact repre-

\footnotetext{
a e-mail: cpinter.livia@uni-obuda.hu (corresponding author)
}

sentation in the complex plane of the dynamic balance between inertia, stiffness, damping and external forces [15].

To overcome the lack of the previously mentioned methods, in this paper the rotating vector solving method, developed for the one degree-of-freedom oscillator, is extended for solving of multi degree-of-freedom systems.

The paper has four sections. In Sect. 2 , the $n$-mass system with $n$ degrees of freedom are physically and mathematically modeled. In Sect. 3 the rotating vector method based on the dynamic balance of internal and external forces is extended for solving $n$ degrees of freedom systems. The relative amplitude and phase angles for bodies in oscillator are calculated. The procedure is applied on vibration of a system modeled with three coupled differential equations (Sect. 4). The amplitudefrequency and phase-frequency relations are studied. The paper ends with Conclusion.

\section{Model of the multi body and multi degree-of-freedom system}

In Fig. 1 the multi body oscillator is plotted. Masses $m_{1}, m_{2}, \ldots, m_{\mathrm{n}}$ are connected with linear springs with rigidity $s_{l}$ and dashpots with linear viscosity and coefficients of damping $k_{l}$ where $l=1,2, \ldots \mathrm{n}$. Connection of mass $m_{1}$ to the fixed body is nonlinear. The elastic 
force in the spring is a nonlinear deflection function, while the damping force is a nonlinear velocity function. The nonlinearity may be of any order given with an integer or non-integer not smaller than 1.

On the mass $m_{1}$ a periodical force $F(t)=F_{0} \sin \left(\omega_{g} t\right)$ acts, where $F_{0}$ and $\omega_{g}$ are the amplitude and the frequency of the excitation force, respectively. Using the principle of D'Alembert, the oscillatory system is mathematically modeled as

$$
\begin{aligned}
m_{1} \ddot{x}_{1} & +s_{1} x_{1}+k_{1} \dot{x}_{1}+s_{\alpha} x_{1}\left|x_{1}\right|^{\alpha-1} \\
& +k_{\beta} \dot{x}_{1}\left|\dot{x}_{1}\right|^{\beta-1}+s_{2}\left(x_{1}-x_{2}\right)+k_{2}\left(\dot{x}_{1}-\dot{x}_{2}\right) \\
= & F_{0} \sin \left(\omega_{g} t\right) \\
m_{l} \ddot{x}_{l}+ & s_{l}\left(x_{l}-x_{l-1}\right)+k_{l}\left(\dot{x}_{l}-\dot{x}_{l-1}\right)+s_{l+1}\left(x_{l}-x_{l+1}\right) \\
& +k_{l+1}\left(\dot{x}_{l}-\dot{x}_{l+1}\right)=0, l=2,3, \ldots n-1 \\
m_{n} \ddot{x}_{n} & +s_{n}\left(x_{n}-x_{n-1}\right)+k_{n}\left(\dot{x}_{n}-\dot{x}_{n-1}\right)=0
\end{aligned}
$$

where $x_{1}, x_{2}, \ldots, x_{\mathrm{n}}$ are position functions, $s_{1}, s_{2}, \ldots$, $s_{\mathrm{n}}$ are coefficients of linear rigidity, $k_{1}, k_{2}, \ldots, k_{\mathrm{n}}$ are coefficients of linear damping, $s_{\alpha}$ is the coefficient of nonlinear rigidity and $k_{\alpha}$ is the coefficient of nonlinear damping. Due to nonlinearity the closed form analytical solution of the system does not exist.

However, for the linear system when $s_{\alpha}=0$ and $k_{\alpha}=0$, when the excitation force $F(t)=F_{0} \sin \left(\omega_{g} t\right)$ has the amplitude $F_{0}$ and the frequency $\omega_{g}$, the particular solution of (1-3) has the form

$$
x_{l}=A_{l} \sin \left(\omega_{g} t+\varphi_{l}\right), \quad l=1,2, \ldots, n
$$

where $A_{l}$ is the unknown amplitude of vibration, and $\varphi_{l}$ is unknown phase angle. For that case, substituting (4) into (1-3) a system of $n$ strong nonlinear algebraic equations are obtained

$$
\begin{aligned}
&-m_{1} A_{1} \omega_{g}^{2} \sin \left(\omega_{g} t+\varphi_{1}\right)+s_{1} A_{1} \sin \left(\omega_{g} t+\varphi_{1}\right) \\
&-k_{1} A_{1} \omega_{g} \cos \left(\omega_{g} t+\varphi_{1}\right) \\
&+s_{2}\left(A_{1} \sin \left(\omega_{g} t+\varphi_{1}\right)-A_{2} \sin \left(\omega_{g} t+\varphi_{1}\right)\right) \\
&-k_{2} \omega_{g}\left(A_{1} \cos \left(\omega_{g} t+\varphi_{1}\right)-A_{2} \cos \left(\omega_{g} t+\varphi_{1}\right)\right) \\
&= F_{0} \sin \left(\omega_{g} t\right), \\
&-m_{l} A_{l} \omega_{g}^{2} \sin \left(\omega_{g} t+\varphi_{l}\right)+s_{l}\left(A_{l} \sin \left(\omega_{g} t+\varphi_{l}\right)\right.\left.\quad-A_{l-1} \sin \left(\omega_{g} t+\varphi_{l-1}\right)\right)-k_{l} \omega_{g}\left(A_{l} \cos \left(\omega_{g} t+\varphi_{l}\right)\right. \\
&\left.-A_{l-1} \cos \left(\omega_{g} t+\varphi_{l-1}\right)\right)+s_{l+1}\left(A_{l} \sin \left(\omega_{g} t+\varphi_{l}\right)\right. \\
&\left.-A_{l+1} \sin \left(\omega_{g} t+\varphi_{l+1}\right)\right) \\
&-k_{l+1} \omega_{g}\left(A_{l} \cos \left(\omega_{g} t+\varphi_{l}\right)\right. \\
&\left.-A_{l+1} \cos \left(\omega_{g} t+\varphi_{l+1}\right)\right)=0, l=2,3, \ldots n-1 \\
&-m_{n} A_{n} \sin \left(\omega_{g} t+\varphi_{n}\right)+s_{n}\left(A_{n} \sin \left(\omega_{g} t+\varphi_{n}\right)\right. \\
&\left.\quad-A_{n-1} \sin \left(\omega_{g} t+\varphi_{n-1}\right)\right)-k_{n} \omega_{g}\left(A_{n} \cos \right. \\
&\left.\quad \times\left(\omega_{g} t+\varphi_{n}\right)-A_{n-1} \cos \left(\omega_{g} t+\varphi_{n-1}\right)\right)=0 .
\end{aligned}
$$

Separating the terms with the same periodic functions (sine and cosine), Eqs. (5-7) transform into $2 n$ algebraic equations with $2 n$ unknown coefficients $A_{l}$ and $\varphi_{l}$ where $l=1,2, \ldots, n$.
Using the exact solutions of (5-7), a solving procedure for the system (1-3) with small nonlinearity is developed.

\section{Method of rotating vector}

For simplification, let us rewrite the Eqs. (1-3) into

$$
\begin{aligned}
& m_{1} \ddot{x}_{1}+s_{1} x_{1}+k_{1} \dot{x}_{1}+s_{\alpha} x_{1}\left|x_{1}\right|^{\alpha-1} \\
& \quad+k_{\beta} \dot{x}_{1}\left|\dot{x}_{1}\right|^{\beta-1}+\sum_{i=2}^{n} m_{i} \ddot{x}_{i} \\
& \quad-F_{0} \exp \left(i \omega_{g} t\right)=0, \\
& m_{l} \ddot{x}_{l}+s_{l} x_{l}+k_{l} \dot{x}_{l}-s_{l} x_{l-1}-k_{l} \dot{x}_{l-1} \\
& \quad+\sum_{i=l+1}^{n} m_{i} \ddot{x}_{i}=0, \quad l=2,3, \ldots n-1 \\
& m_{n} \ddot{x}_{n}+s_{n} x_{n}+k_{n} \dot{x}_{n} \\
& \left.\quad-s_{n} x_{n-1}-k_{n} \dot{x}_{n-1}\right)=0,
\end{aligned}
$$

where $F_{0} \exp (i \Omega t)$ is a periodical excitation force and $i=\sqrt{-1}$ is the imaginary unit. Introducing rotating vectors with constant amplitude $A_{s}$ and phase $\theta_{s}$ :

$$
x_{l}=A_{l} \exp i\left(\omega_{g} t+\varphi_{l}\right)
$$

and corresponding time derivatives

$$
\begin{aligned}
\dot{x}_{l} & =A_{l} i \omega_{g} \exp i\left(\omega_{g} t+\varphi_{l}\right) \\
& =A_{;} \omega_{g} \exp i\left(\omega_{g} t+\varphi_{l}+\frac{\pi}{2}\right), \\
\ddot{x}_{l} & =A_{l} \omega_{g}^{2} i^{2} \exp i\left(\omega_{g} t+\varphi_{l}\right) \\
& =A_{l} \omega_{g}^{2} \exp i\left(\omega_{g} t+\varphi_{l}+\pi\right) \\
& =-A_{l} \omega_{g}^{2} \exp i\left(\omega_{g} t+\varphi_{l}\right)
\end{aligned}
$$

Eqs. (8-10) transform into

$$
\begin{aligned}
& A_{1} \exp i\left(\omega_{g} t+\varphi_{1}\right)\left(-m_{1} \omega_{g}^{2}+s_{1}+k_{1} \omega_{g} \exp \left(\frac{i \pi}{2}\right)\right) \\
& +s_{\alpha} A_{1} \exp i\left(\omega_{g} t+\varphi_{1}\right)\left|A_{1} \exp i\left(\omega_{g} t+\varphi_{1}\right)\right|^{\alpha-1}+ \\
& \quad+k_{\beta} A_{1} \omega_{g} \exp i\left(\omega_{g} t+\varphi_{1}+\frac{\pi}{2}\right) \\
& \left|A_{1} \omega_{g} \exp i\left(\omega_{g} t+\varphi_{1}+\frac{\pi}{2}\right)\right|^{\beta-1} \\
& \quad-\sum_{i=2}^{n} m_{i} \omega_{g}^{2} A_{i} \exp i\left(\omega_{g} t+\varphi_{i}\right)=F_{0} \exp i\left(\omega_{g} t\right) \quad(14) \\
& A_{l} \exp \left(i \varphi_{l}\right)\left(-m_{l} \omega_{g}^{2}+s_{l}+k_{l} \omega_{g} \exp \left(\frac{\mathrm{i} \pi}{2}\right)\right) \\
& \quad-A_{l-1} \exp \left(i \varphi_{l-1}\right)\left(s_{l}-k_{l} \omega_{g} \exp \left(\frac{i \pi}{2}\right)\right) \\
& -\sum_{i=l+1}^{n} m_{i} A_{i} \omega_{g}^{2} \exp \left(i \varphi_{i}\right)=0, \quad s=2,3, \ldots n-1
\end{aligned}
$$




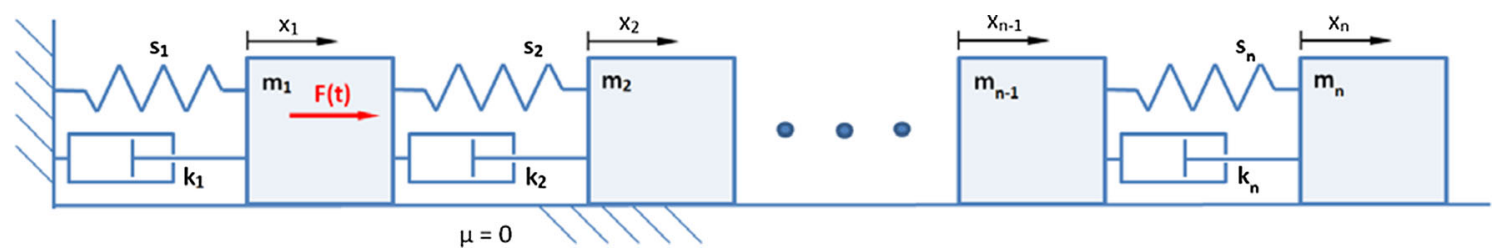

Fig. 1 Model of the multi body oscillator

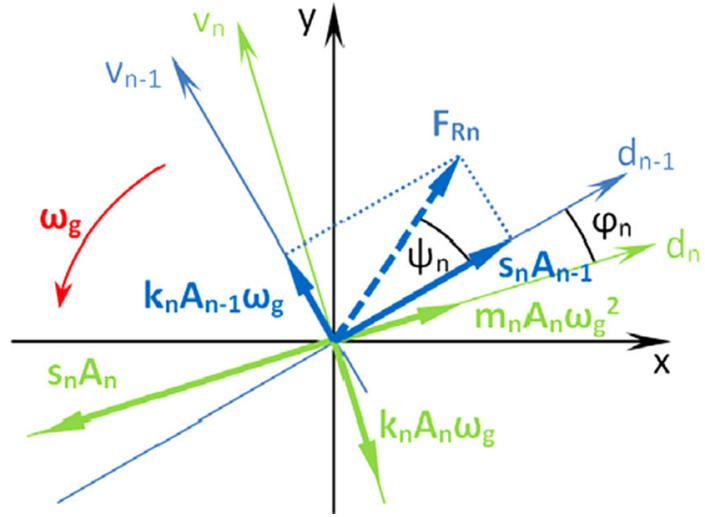

Fig. 2 Rotating vectors acting on the mass $m_{n}$

$$
\begin{gathered}
A_{n} \exp \left(i \varphi_{n}\right)\left(-m_{n} \omega_{g}^{2}+s_{n}+k_{n} \omega_{g} \exp \left(\frac{i \pi}{2}\right)\right) \\
-\exp \left(i \varphi_{n-1}\right)\left(s_{n}+k_{n} \omega_{g} \exp \left(\frac{i \pi}{2}\right)\right)=0
\end{gathered}
$$

To solve the system of Eqs. (14-16) we start with the equation (16) which considers the motion of the mass $m_{n}$ which is the last in the system.

\subsection{Mass $m_{n}$}

In Fig. 2 the rotating vectors for mass $m_{n}$ in the $x-$ $y$ plane complex plane are plotted. Namely, using the relation for the complex number $z=x+i y, x$ and $y$ represent the real and the imaginary value. The plane is assumed to rotate with angular velocity $\omega_{g}$ which corresponds to the excitation frequency.

In the $x-y$ plane, the relation (16) has five terms: one, which represents the inertial force of mass $m_{n}$, and two stiffness and two damping terms in the connection between masses $m_{n}$ and the previous mass $m_{n-1}$.

For simplification, the last two terms of (16) are summarized and substituted with one force $F_{\mathrm{Rn}}$ with angle position $\psi_{n}$ :

$$
F_{\mathrm{Rn}}=A_{n-1} \sqrt{s_{n}^{2}+k_{n}^{2} \omega_{g}^{2}}, \tan \left(\psi_{n}\right)=\frac{k_{n} \omega_{g}}{s_{n}} .
$$

Introducing (17) $)_{1}$ into (16) and projecting the relation on the displacement and velocity axis $d_{n}$ and $v_{n}$ of the mass $m_{\mathrm{n}}$ in the $x-y$ plane, we have

$$
s_{n} A_{n}-m_{n} A_{n} \omega_{g}^{2}-F_{\mathrm{Rn}} \cos \left(\psi_{n}+\varphi_{n}\right)=0,
$$

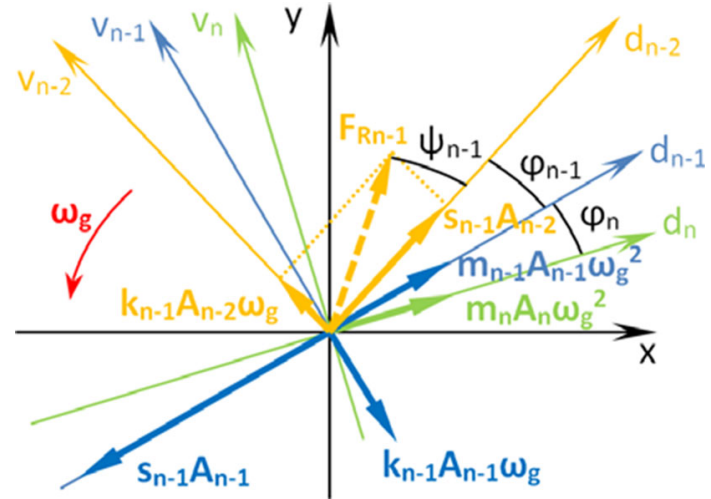

Fig. 3 Rotating vectors of the mass $m_{l}$

$$
k_{n} A_{n} \omega_{g}-F_{\mathrm{Rn}} \sin \left(\psi_{n}+\varphi_{n}\right)=0 .
$$

Rewritting (18) and (19) we obtain

$$
\begin{aligned}
\sqrt{s_{n}^{2}+k_{n}^{2} \omega_{g}^{2}} \cos \left(\psi_{n}+\varphi_{n}\right) & =\frac{A_{n}}{A_{n-1}} K_{n}, \\
\sqrt{s_{n}^{2}+k_{n}^{2} \omega_{g}^{2}} \sin \left(\psi_{n}+\varphi_{n}\right) & =\frac{A_{n}}{A_{n-1}} S_{n},
\end{aligned}
$$

where

$$
K_{n}=s_{n}-m_{n} \omega_{g}^{2}, S_{n}=k_{n} \omega_{g}
$$

After some modification equations the amplitude ratio for masses $m_{n-1}$ and $m_{n}$ and the phase angle $\varphi_{n}$ are obtained

$$
\begin{aligned}
\frac{A_{n}}{A_{n-1}} & =q_{n}=\sqrt{\frac{s_{n}^{2}+k_{n}^{2} \omega_{g}^{2}}{K_{n}^{2}+S_{n}^{2}}} \\
\varphi_{n} & =\tan ^{-1}\left(\frac{S_{n}}{K_{n}}\right)-\tan ^{-1}\left(\frac{k_{n} \omega_{g}}{s_{n}}\right)
\end{aligned}
$$

\subsection{Mass $m_{l}$ where $l=2,3, \ldots n-1$}

On any of mass $m_{l}$ which is between the first $m_{1}$ and the last $m_{n}$ mass the following forces act: inertial forces of all masses from $m_{n}$ up to $m_{l}$, and the stiffness and damping forces in the connection between the mass $m_{l}$ and $m_{l-1}$.

According to (15) the rotating vectors for $m_{l}$ are plotted (see Fig. 3).

As it was suggested in the previous section, two vectors $A_{l-1} \exp \left(i \varphi_{l-1}\right)\left(-s_{l}\right)$ and $A_{l-1} \exp \left(i \varphi_{l-1}+\frac{i \pi}{2}\right)$ 
$\left(-k_{l} \omega_{g}\right)$ in (15) are substituted with only one force. In Fig. 4a, the result of adding of two terms is plotted, i.e.,

$$
F_{R l}=A_{l-1} \sqrt{s_{l}^{2}+k_{l}^{2} \omega_{g}^{2}}, \tan \left(\psi_{l}\right)=\frac{k_{l} \omega_{g}}{s_{l}} .
$$

Using (24) the Eq. (15) is projected on the displacement and velocity axes, $d_{l}$ and $v_{l}$ of the mass $m_{l}$ in the complex plane (see Fig. 3) and we obtain

$$
\begin{gathered}
A_{l}\left(-m_{l} \omega_{g}^{2}+s_{l}\right)+F_{\mathrm{RL}} \cos \left(\psi_{l}+\varphi_{l}\right) \\
-\sum_{i=l+1}^{n} m_{i} A_{i} \omega_{g}^{2} \cos \left(\sum_{j=l+1}^{i} \varphi_{j}\right)=0, \\
A_{l} k_{l} \omega_{g}-F_{\mathrm{RL}} \sin \left(\psi_{l}+\varphi_{l}\right) \\
-\sum_{i=l+1}^{n} m_{i} A_{i} \omega_{g}^{2} \sin \left(\sum_{j=l+1}^{i} \varphi_{j}\right)=0 \\
l=2,3, \ldots n-1
\end{gathered}
$$

Substituting (24) into (15) and (26) and after some modification it is

$$
\begin{gathered}
\sqrt{s_{l}^{2}+k_{l}^{2} \omega_{g}^{2}} \cos \left(\psi_{l}+\varphi_{l}\right)=\frac{A_{l}}{A_{l-1}} K_{l}, \\
\sqrt{s_{l}^{2}+k_{l}^{2} \omega_{g}^{2}} \sin \left(\psi_{l}+\varphi_{l}\right)=\frac{A_{l}}{A_{l-1}} S_{l},
\end{gathered}
$$

where

$$
\begin{aligned}
K_{l}= & \left(-m_{l} \omega_{g}^{2}+s_{l}\right) \\
& -\sum_{i=l+1}^{n} m_{i} \omega_{g}^{2}\left(\prod_{j=l+1}^{i} q_{j}\right) \cos \left(\sum_{j=l+1}^{i} \varphi_{j}\right), \\
S_{l}= & k_{l} \omega_{g}-\sum_{i=l+1}^{n} m_{i} \omega_{g}^{2}\left(\prod_{j=l+1}^{i} q_{j}\right) \\
& \sin \left(\sum_{j=l+1}^{i} \varphi_{j}\right) \\
q_{j}= & \frac{A_{j}}{A_{j-1}},
\end{aligned}
$$

Finally, the amplitude of vibration ratio for masses $m_{l}$ and $m_{l+1}$ and the phase angle $\varphi_{l}$ are obtained

$$
\begin{aligned}
& \frac{A_{l}}{A_{l-1}}=q_{l}=\sqrt{\frac{s_{l}^{2}+k_{l}^{2} \omega_{g}^{2}}{K_{l}^{2}+S_{l}^{2}}}, \\
& \varphi_{l}=\tan ^{-1}\left(\frac{S_{l}}{K_{l}}\right)-\tan ^{-1}\left(\frac{k_{l} \omega_{g}}{s_{l}}\right) .
\end{aligned}
$$

\subsection{Mass $m_{1}$}

Finally, the dynamic balance of forces for mass $m_{1}$ is given with equation (14). It is seen that the nonlinear terms are with angle position given with trigonometric functions $\cos \left(\omega_{g} t+\varphi_{1}\right)\left|\cos \left(\omega_{g} t+\varphi_{1}\right)\right|^{\alpha-1}$ and $\sin \left(\omega_{g} t+\varphi_{1}\right)\left|\sin \left(\omega_{g} t+\varphi_{1}\right)\right|^{\alpha-1}$ and also $\cos \left(\omega_{g} t+\right.$ $\left.\varphi_{1}\right)\left|\cos \left(\omega_{g} t+\varphi_{1}\right)\right|^{\beta-1}$ and $\sin \left(\omega_{g} t+\varphi_{1}\right) \mid \sin \left(\omega_{g} t+\varphi_{1}\right)$ $\left.\right|^{\beta-1}$. Let us introduce the Fourier series for $\mid \cos \left(\omega_{g} t\right.$ $\left.+\varphi_{1}\right)\left.\right|^{\alpha-1}$ and $\left|\sin \left(\omega_{g} t+\varphi_{1}\right)\right|^{\alpha-1}$, i.e., $\mid \cos \left(\omega_{g} t\right.$ $\left.+\varphi_{1}\right)\left.\right|^{\beta-1}$ and $\left|\sin \left(\omega_{g} t+\varphi_{1}\right)\right|^{\beta-1}$ using the relation [16]

$$
\begin{aligned}
& (\cos (\theta))^{p}=\sum_{i=0}^{\infty} a_{i} \cos (i \theta), \\
& (\sin (\theta))^{p}=\sum_{i=0}^{\infty} b_{i} \sin (i \theta) .
\end{aligned}
$$

where

$$
\begin{aligned}
a_{i} & =\left(\frac{4}{\pi}\right) \int_{0}^{\pi / 2}(\cos (\theta))^{p} \cos (i \theta) \mathrm{d} \theta, \\
b_{i} & =\left(\frac{4}{\pi}\right) \int_{0}^{\pi / 2}(\sin (\theta))^{p} \sin (i \theta) \mathrm{d} \theta
\end{aligned}
$$

and

$$
\int_{0}^{\pi / 2}(\cos (\theta))^{p} \cos (i \theta) \mathrm{d} \theta=\frac{\pi}{2^{p+1}(p+1) B\left(\frac{p+i+2}{2}, \frac{p-i+2}{2}\right)},
$$

where $\mathrm{B}(p, q)$ is the beta function [10].

Assuming the first term of the series expansions (33) we have

$$
\begin{aligned}
& \cos (\theta)|\cos (\theta)|^{p} \\
& \quad=a_{0} \cos (\theta)+\cdots, \sin (\theta)|\sin (\theta)|^{p}=b_{0} \sin (\theta)+\cdots
\end{aligned}
$$

Introducing the first approximation (35) into (14), we obtain the system of vectors rotating with angular velocity $\omega_{\mathrm{g}}$. In Fig. 4 the system after approximation is plotted.

Projecting the Eq. (14) on the axis $d_{1}$ and $v_{1}$ in complex plane (see Fig. 4) it is

$$
\begin{aligned}
& A_{1}\left(-m_{1} \omega_{g}^{2}+s_{1}\right)+a_{0} s_{\alpha} A_{1} \\
& -\sum_{i=2}^{n} m_{i} \omega_{g}^{2} A_{i} \cos \left(\sum_{j=2}^{i} \varphi_{j}\right) \\
& =F_{0} \cos \left(\varphi_{1}\right) \\
& A_{1} k_{1} \omega_{g}+b_{0} k_{\beta} A_{1} \omega_{g}
\end{aligned}
$$




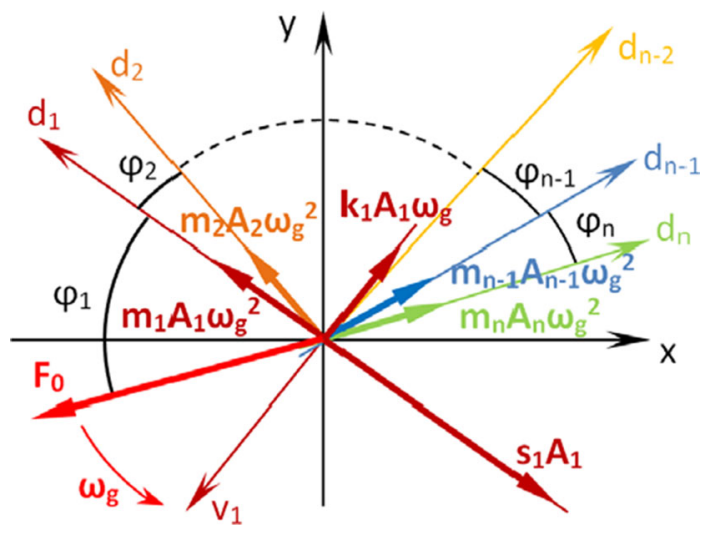

Fig. 4 Rotating vectors of the mass $m_{1}$ in complex plane

$$
\begin{aligned}
& -\sum_{i=2}^{n} m_{i} \omega_{g}^{2} A_{i} \sin \left(\sum_{j=2}^{i} \varphi_{j}\right) \\
= & F_{0} \sin \left(\varphi_{1}\right)
\end{aligned}
$$

i.e.,

$$
\begin{aligned}
& A_{1} K_{1}=F_{0} \cos \left(\varphi_{1}\right) \\
& A_{1} S_{1}=F_{0} \sin \left(\varphi_{1}\right)
\end{aligned}
$$

where

$$
\begin{aligned}
K_{1}= & \left(-m_{1} \omega_{g}^{2}+s_{1}\right)+a_{0} s_{\alpha} \\
& -\sum_{i=2}^{n} m_{i} \omega_{g}^{2}\left(\prod_{j=2}^{i} q_{j}\right) \cos \left(\sum_{j=2}^{i} \varphi_{j}\right), \\
S_{1}= & {\left[k_{1} \omega_{g}+b_{0} k_{\beta} \omega_{g}\right.} \\
& \left.-\sum_{i=2}^{n} m_{i} \omega_{g}^{2}\left(\prod_{j=2}^{i} q_{j}\right) \sin \left(\sum_{j=2}^{i} \varphi_{j}\right)\right]
\end{aligned}
$$

Finally, according to (38) the approximate solution for the amplitude and phase of vibration of the mass 1 is
Using the values (41) and substituting into (32) and (23)amplitudes and phases of vibration of masses $2,3, \ldots, n$ are obtained.

\section{Vibration of the three-degree-of freedom system}

Let us consider the three mass oscillatory system with three degrees of freedom. The elastic and damping properties of the spring and dashpot connected with the mass $m_{1}$ are nonlinear. The order of nonlinearity is assumed to be $3 / 2$. Connection between masses $m_{2}$ and $m_{3}$ are linear. On the mass $m_{1}$ the excitation force $F_{0} \sin \left(\omega_{g} t\right)$ acts, where $F_{0}$ and $\omega_{g}$ are amplitude and frequency, respectively. Motion of the three mass system is described with coupled weakly nonlinear differential equations

$$
\begin{aligned}
m_{1} \ddot{x}_{1} & +s_{1} x_{1}+k_{1} \dot{x}_{1}+s_{\alpha} x_{1}\left|x_{1}\right|^{1 / 2} \\
& +k_{\beta} \dot{x}_{1}\left|\dot{x}_{1}\right|^{1 / 2}+s_{2}\left(x_{1}-x_{2}\right)+k_{2}\left(\dot{x}_{1}-\dot{x}_{2}\right) \\
= & F_{0} \sin \left(\omega_{g} t\right) \\
m_{2} \ddot{x}_{2} & +s_{2}\left(x_{2}-x_{1}\right)+k_{2}\left(\dot{x}_{2}-\dot{x}_{1}\right)+s_{3}\left(x_{2}-x_{3}\right) \\
& +k_{3}\left(\dot{x}_{2}-\dot{x}_{3}\right)=0 \\
m_{3} \ddot{x}_{3} & +s_{3}\left(x_{3}-x_{2}\right) \\
& +k_{3}\left(\dot{x}_{3}-\dot{x}_{2}\right)=0
\end{aligned}
$$

where $s_{i}$ are coefficients of linear stiffness, $s_{\alpha} \ll 1$ is the coefficient of small nonlinear stiffness, $k_{i}$ are coefficients of linear damping, $k_{\beta}$ is the coefficient of small nonlinear damping and $i=1,2,3$. For the system with weak nonlinearities, the aforementioned approximate solving method of the problem is applied.

Thus, the relative amplitudes of vibration and the phase angles are due to (21) and (32)

$$
\begin{aligned}
& \frac{A_{3}}{A_{2}}=\sqrt{\frac{s_{3}^{2}+k_{3}^{2} \omega_{g}^{2}}{\left(s_{3}-m_{3} \omega_{g}^{2}\right)^{2}+k_{3}^{2} \omega_{g}^{2}}}=q_{3}, \\
& \varphi_{3}=\tan ^{-1}\left(\frac{k_{3} \omega_{g}}{s_{3}-m_{3} \omega_{g}^{2}}\right)-\tan ^{-1}\left(\frac{k_{3} \omega_{g}}{s_{3}}\right),
\end{aligned}
$$

and

$$
\begin{aligned}
\frac{A_{2}}{A_{1}} & =\sqrt{\frac{s_{2}^{2}+k_{2}^{2} \omega_{g}^{2}}{\left(s_{2}-m_{2} \omega_{g}^{2}-m_{3} q_{3} \omega_{g}^{2} \cos \left(\varphi_{3}\right)\right)^{2}+\left(k_{2} \omega_{g+} m_{3} q_{3} \omega_{g}^{2} \sin \left(\varphi_{3}\right)\right)^{2}}}=q_{2}, \\
\varphi_{2} & =\tan ^{-1}\left(Q_{2}\right)-\tan ^{-1}\left(\frac{k_{2} \omega_{g}}{s_{2}}\right),
\end{aligned}
$$

$$
\begin{aligned}
& A_{1}=\frac{F_{0}}{\sqrt{K_{1}^{2}+S_{1}^{2}}}, \\
& \varphi_{1}=\tan ^{-1}\left(\frac{S_{1}}{K_{1}}\right)
\end{aligned}
$$


where

$$
\begin{aligned}
Q_{2} & =\tan \left(\psi_{2}+\varphi_{2}\right) \\
& =\frac{k_{2} \omega_{g}+m_{3} \omega_{g}^{2} q_{3} \sin \left(\varphi_{3}\right)}{s_{2}-m_{2} \omega_{g}^{2}-m_{3} \omega_{g}^{2} q_{3} \cos \left(\varphi_{3}\right)} .
\end{aligned}
$$

Introducing the frequency $\omega_{i}$ damping $\zeta_{i}$ and mass $\mu$ parameters

$$
\begin{aligned}
& \omega_{i}=\sqrt{\frac{s_{i}}{m_{i}}}, \quad \zeta_{i}=\sqrt{\frac{k_{i}^{2}}{m_{i} s_{i}}}, \quad \mu_{i-1}=\frac{m_{i}}{m_{i-1}}, \\
& s=\frac{s_{\alpha}}{s_{1}}, \quad \zeta=\sqrt{\frac{k_{\beta}^{2}}{m_{1} s_{1}}},
\end{aligned}
$$

where $i=1,2,3$, the amplitude and phase relations (45) and (46) with (47) transform into

$$
\begin{aligned}
\frac{A_{3}}{A_{2}}= & \sqrt{\frac{1+\zeta_{3}^{2}\left(\frac{\omega_{g}^{2}}{\omega_{3}^{2}}\right)}{\left(1-\left(\frac{\omega_{g}^{2}}{\omega_{3}^{2}}\right)\right)^{2}+\zeta_{3}^{2}\left(\frac{\omega_{g}^{2}}{\omega_{3}^{2}}\right)}}, \\
\varphi_{3}= & \tan ^{-1}\left(\frac{\zeta_{3}\left(\frac{\omega_{g}}{\omega_{3}}\right)}{1-\left(\frac{\omega_{g}^{2}}{\omega_{3}^{2}}\right)}\right) \\
& -\tan ^{-1}\left(\frac{\zeta_{3} \omega_{g}}{\omega_{3}}\right)
\end{aligned}
$$

and the frequency ratio is smaller than 1 . In Fig. $5 b$ it is shown that the phase angle is increasing with the damping coefficient $\zeta_{3}$. For the case when damping is omitted and $\zeta_{3}=0$, the phase angle is zero.

In Fig. 6 the ratio of the amplitude of vibration $A_{2}$ of the mass $m_{2}$ to amplitude of vibration of $A_{1}$ of the mass $m_{1}$ as the function of the excitation frequency $\omega_{\mathrm{g}}(51)$ is plotted. The $A_{2} / A_{1}$ amplitude ratio is shown for the resonant value of the amplitude $A_{3}$ when $\omega_{\mathrm{g}} / \omega_{3}=0.995$ and $\zeta_{3}=0.5$. The diagrams are plotted for various damping parameter $\zeta_{2}$. For the same parameter values the phase angle $\varphi_{2}$ as functions of $\omega_{\mathrm{g}} / \omega_{2}$, for various damping parameter $\zeta_{2}(52)$ is plotted in Fig. $6 \mathrm{~b}$. It is obtained that the resonant amplitude decreases with increase of the damping parameter $\zeta_{2}$ The resonant amplitude moves toward smaller values of $\omega_{\mathrm{g}}$ by increasing of $\zeta_{2}$. Even the coefficient $\zeta_{2}$ is zero, the phase angle exist.

Substituting (35) into (42) it is seen that the mass $m_{1}$ is under influence of linear and nonlinear forces. Assuming the first term of Fourier transformation [16] i.e., $\cos (\theta)|\cos (\theta)|^{1 / 2}=0.71317 \cos (\theta)$ and $\sin (\theta)|\sin (\theta)|^{1 / 2}=0.71317 \cos (\theta)$ and neglecting the other terms as small values, the approximate values for the relations (39) and (40) are obtained

$$
\begin{aligned}
K_{1}= & s_{1}-m_{1} \omega_{g}^{2}+0.71317 s_{\alpha} \\
& -m_{2} \omega_{g}^{2} q_{2} \cos \left(\varphi_{2}\right) \\
& -m_{3} \omega_{g}^{2} q_{2} q_{3} \cos \left(\varphi_{2}+\varphi_{3}\right),
\end{aligned}
$$

$$
\frac{A_{2}}{A_{1}}=\sqrt{\frac{1+\zeta_{2}^{2}\left(\frac{\omega_{g}^{2}}{\omega_{2}^{2}}\right)}{\left(1-\left(\frac{\omega_{g}^{2}}{\omega_{2}^{2}}\right)-\mu_{2} q_{3}\left(\frac{\omega_{g}^{2}}{\omega_{2}^{2}}\right) \cos \left(\varphi_{3}\right)\right)^{2}+\left(\frac{\omega_{g}}{\omega_{2}} \zeta_{2}+\mu_{2} q_{3}\left(\frac{\omega_{g}^{2}}{\omega_{2}^{2}}\right) \sin \left(\varphi_{3}\right)\right)^{2}}}
$$

$$
\begin{aligned}
& \varphi_{2}=\tan ^{-1}\left(Q_{2}\right)-\tan ^{-1}\left(\frac{\omega_{g}}{\omega_{2}} \zeta_{2}\right), \\
& Q_{2}=\tan \left(\psi_{2}+\varphi_{2}\right)=\frac{\frac{\omega_{g}}{\omega_{2}} \zeta_{2}+\mu_{2}\left(\frac{\omega_{g}^{2}}{\omega_{2}^{2}}\right) q_{3} \sin \left(\varphi_{3}\right)}{1-\left(\frac{\omega_{g}^{2}}{\omega_{2}^{2}}\right)-\mu_{2}\left(\frac{\omega_{g}^{2}}{\omega_{2}^{2}}\right) q_{3} \cos \left(\varphi_{3}\right)}
\end{aligned}
$$

Using (49-52) the amplitude-frequency and phasefrequency diagrams are plotted.

In Fig. 5a the diagram of amplitude ratio $A_{3} / A_{2}$ (49) and in Fig. 5b of the phase angle $\varphi_{3}(50)$ as function of $\omega_{\mathrm{g}} / \omega_{3}$, for various damping parameter $\zeta_{3}$ are plotted.

In Fig. $5 \mathrm{a}$ the relation between the amplitude of vibration $A_{3}$ of the mass $m_{3}$ and the amplitude $A_{2}$ of the mass $m_{2}$ depending on the excitation frequency $\omega_{\mathrm{g}}$ is plotted. It is obvious that the resonant frequency and amplitude depend on the damping coefficient $\zeta_{3}$ : the higher is the damping the resonant amplitude is lower

$$
\begin{aligned}
S_{1}= & k_{1} \omega_{g}+0.71317 k_{\beta} \omega_{g} \\
& -m_{2} \omega_{g}^{2} q_{2} \sin \left(\varphi_{2}\right) \\
& -m_{3} \omega_{g}^{2} q_{2} q_{3} \sin \left(\varphi_{2}+\varphi_{3}\right) .
\end{aligned}
$$

Using the dimensionless parameters (48) it is

$$
K_{1}=m_{1} \omega_{1}^{2} K, S_{1}=m_{1} \omega_{1}^{2} S,
$$

where

$$
\begin{aligned}
K= & 1+0.71317 s-\frac{\omega_{g}^{2}}{\omega_{1}^{2}}-\mu_{1} \frac{\omega_{g}^{2}}{\omega_{1}^{2}} q_{2} \cos \left(\varphi_{2}\right) \\
& -\mu_{1} \mu_{2} \frac{\omega_{g}^{2}}{\omega_{1}^{2}} q_{2} q_{3} \cos \left(\varphi_{2}+\varphi_{3}\right),
\end{aligned}
$$



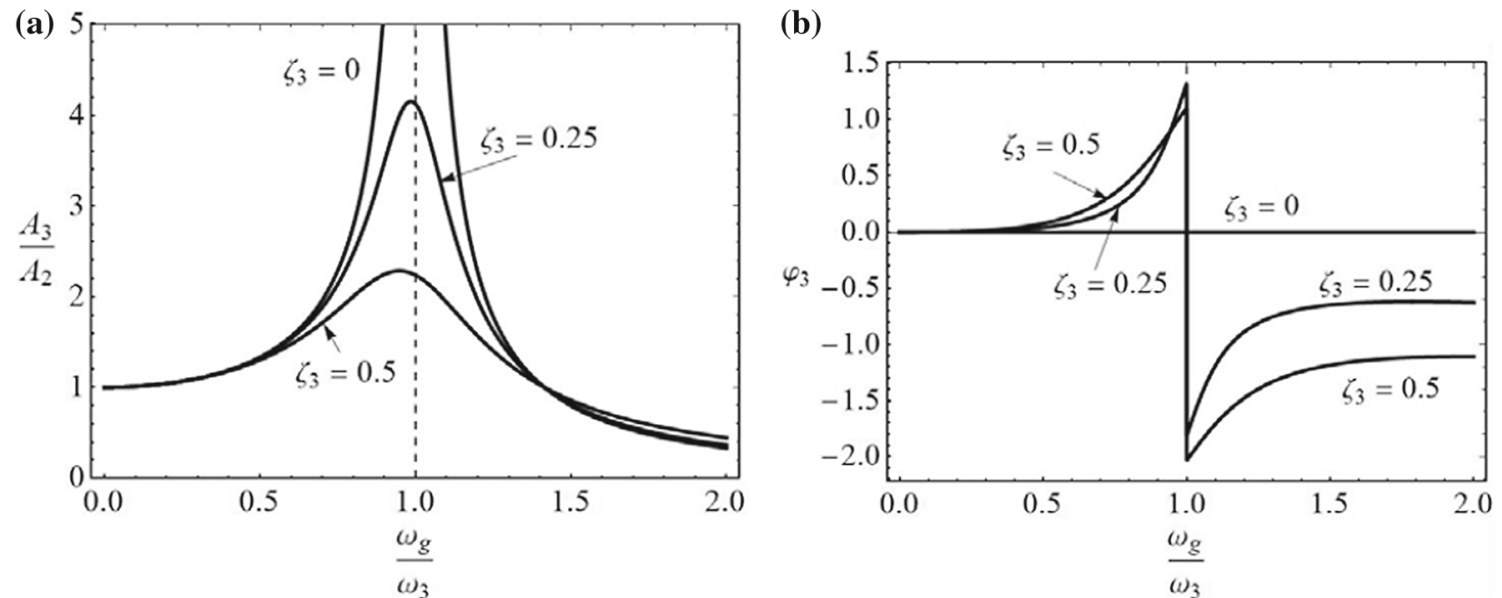

Fig. 5 a $A_{3} / A_{2}-\omega_{\mathrm{g}} / \omega_{3}$ diagrams for various $\zeta_{3} ; \mathbf{b} \varphi_{3}-\omega_{\mathrm{g}} / \omega_{3}$ diagrams for various $\zeta_{3}$
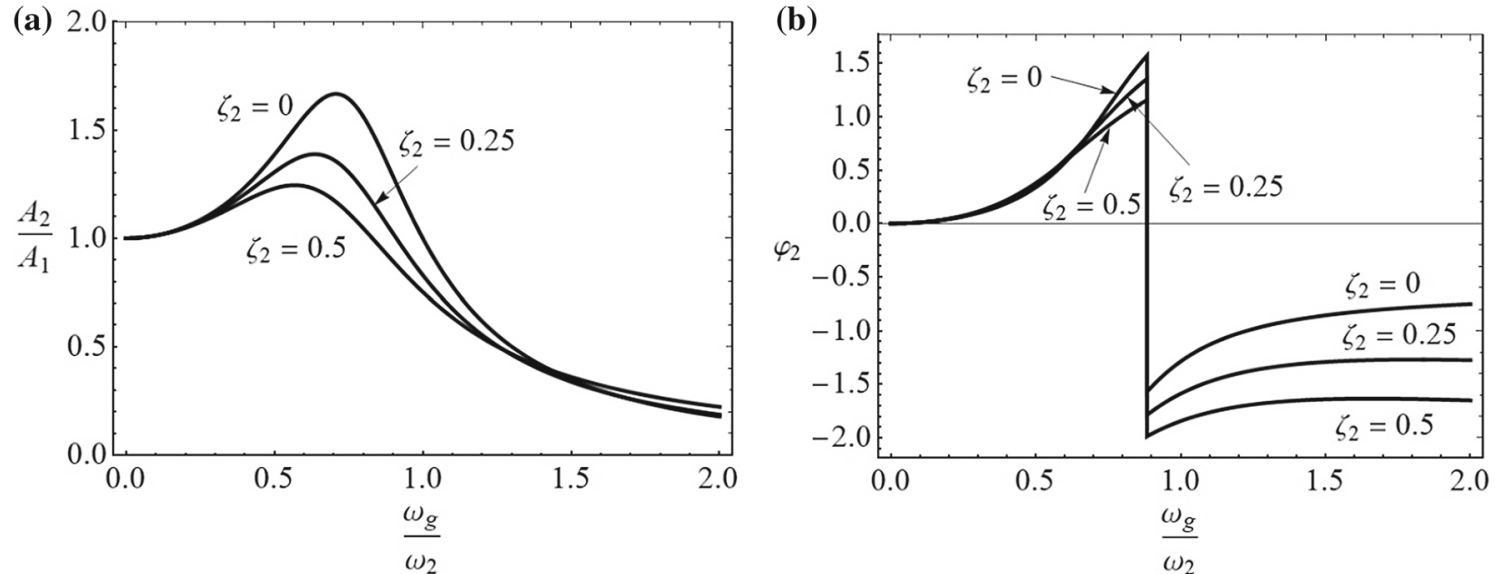

Fig. 6 a $A_{2} / A_{1}-\omega_{\mathrm{g}} / \omega_{2}$ diagrams for various $\zeta_{2} ; \mathbf{b} \varphi_{2}-\omega_{\mathrm{g}} / \omega_{2}$ diagrams for various $\zeta_{2}$

$$
\begin{aligned}
S= & \zeta_{1} \frac{\omega_{g}}{\omega_{1}}+0.71317 \zeta \frac{\omega_{g}}{\omega_{1}}-\mu_{1} \frac{\omega_{g}^{2}}{\omega_{1}^{2}} q_{2} \sin \left(\varphi_{2}\right) \\
& -\mu_{1} \mu_{2} \frac{\omega_{g}^{2}}{\omega_{1}^{2}} q_{2} q_{3} \sin \left(\varphi_{2}+\varphi_{3}\right) .
\end{aligned}
$$

Substituting (55) into (41) the amplitude and phase relations are obtained

$$
\begin{array}{r}
A_{1}=\frac{F_{0}}{m_{1} \omega_{1}^{2} Q}, \\
\varphi_{1}=\tan ^{-1}\left(\frac{S}{K}\right),
\end{array}
$$

where

$$
Q=\sqrt{K^{2}+S^{2}}
$$

In Figs. 7 and 8, the $\frac{F_{0}}{m_{1} \omega_{1}^{2} A_{1}}=Q$ diagrams, as the function of the frequency ratio $\frac{\omega_{g}}{\omega_{1}}$ for various values of nonlinear stiffness $s$ and damping $\zeta_{1}$, are plotted. In Fig. 7a the diagrams of ratio of the resonant amplitude of vibration of the flexible beam $\mathrm{A}_{1}$ and the amplitude of excitation $F_{0}$ i.e. $1 / Q$, as the function of the excitation frequency for various damping parameters are plotted. For $\omega_{\mathrm{g}} / \omega_{2}=0.52$ and $\zeta_{2}=0.5$ maximum of the relation $1 / \mathrm{Q}$ for $\zeta_{1}=0$ is 1 at $\omega_{\mathrm{g}} / \omega_{1}=0.55$ and the maximum increases with $\zeta_{1}$. The phase angle has the tendency of decrease with $\omega_{\mathrm{g}} / \omega_{1}$.

In Fig. 8 the influence on the nonlinear rigidity on the vibration properties of the system is shown. Increasing the coefficient of the nonlinear stiffness the maximal amplitude of vibration decreases and its position tends to $\omega_{\mathrm{g}} / \omega_{1}=1$ (Fig. 8a). In Fig. 8b the phase angle variation according to nonlinear property is shown. Maximum of the phase angle does not depend on s, but its position tends to move from $\omega_{\mathrm{g}} / \omega_{1}=1$ with increasing of $s$. It is worth to be said that the influence of nonlinear rigidity on the amplitude and phase characteristics can be omitted for $\omega_{\mathrm{g}} / \omega_{1}>>1$.

Substituting (58) into (50) and (49), we obtain

$$
\begin{aligned}
& A_{2}=\frac{F_{0} q_{2}}{m_{1} \omega_{1}^{2} Q} \\
& A_{3}=\frac{F_{0} q_{2} q_{3}}{m_{1} \omega_{1}^{2} Q}
\end{aligned}
$$



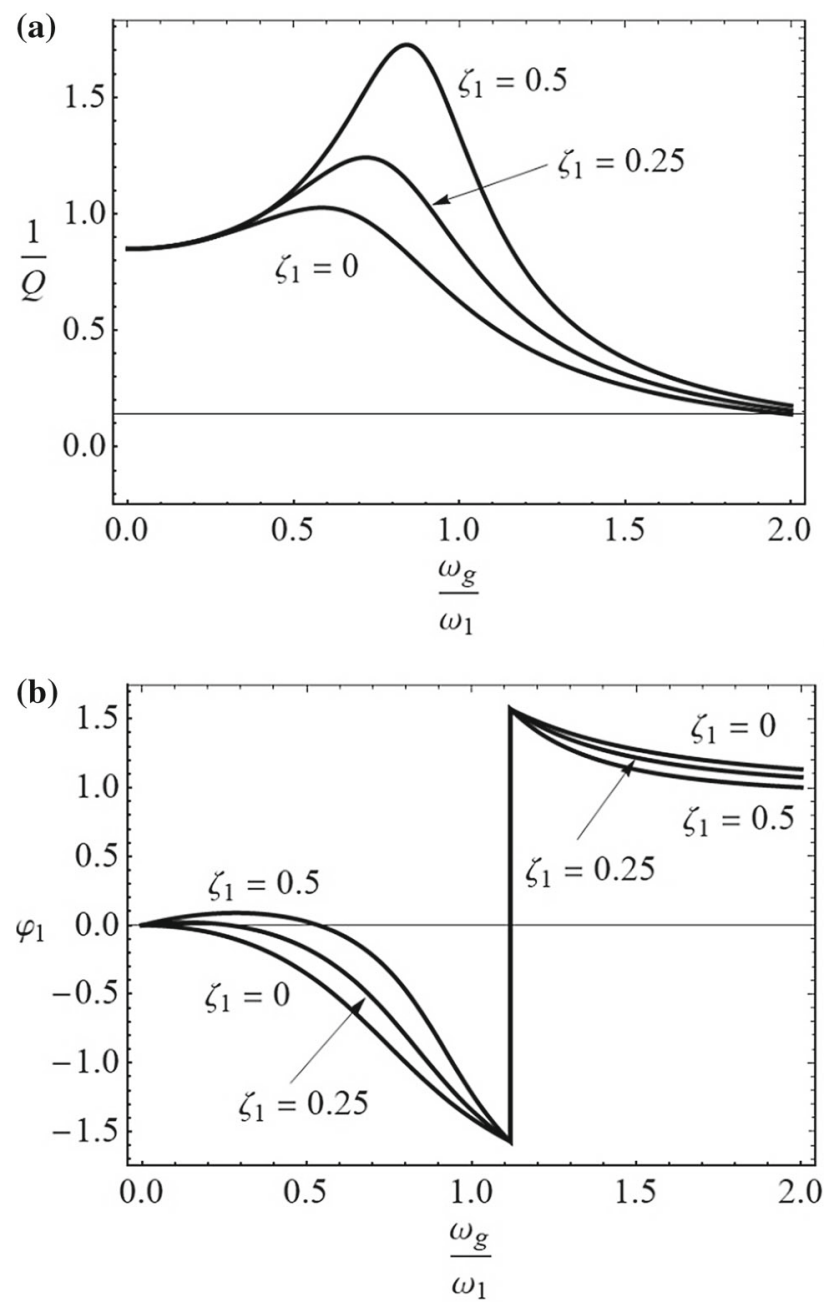

Fig. 7 a $1 / Q-\omega_{\mathrm{g}} / \omega_{1}$ diagrams for various $\zeta_{1} ; \mathbf{b} \varphi_{1}-\omega_{\mathrm{g}} / \omega_{3}$ diagrams for various $\zeta_{1}$

The relations (61) represent the approximate amplitudes of vibration of masses $m_{2}$ and $m_{3}$ according to the amplitude of vibration of the mass $m_{1}$. In the expressions (61), the influence of the nonlinear stiffness property is incorporated by correcting the amplitudes according to $A_{1}$. However, the influence of the phase angle of the first mass has negligible influence on the corresponding phase angles of the masses $m_{2}$ and $m_{3}$.

Numerical example

Let us consider a system with three equal masses $m_{1}=m_{2}=m_{3}=1 \mathrm{~g}$, with equal linear stiffness coefficients $s_{1}=s_{2}=s_{3}=1 \mathrm{~g} / \mathrm{s}^{2}$ and without damping. The equations of oscillatory motion are

$$
\begin{aligned}
& \ddot{x}_{1}+x_{1}+s_{\alpha} x_{1}\left|x_{1}\right|^{1 / 2}+\left(x_{1}-x_{2}\right)=\cos (2 t), \\
& \ddot{x}_{2}+\left(x_{2}-x_{1}\right)+\left(x_{2}-x_{3}\right)=0, \\
& \ddot{x}_{3}+\left(x_{3}-x_{2}\right)=0,
\end{aligned}
$$

where the excitation amplitude and frequency are $F_{0}=$ $1 \mathrm{~g} \mathrm{~cm} / \mathrm{s}^{2}$ and $\omega_{g}=2 \mathrm{~s}^{-1}$. The equations $(63-65)$ are solved analytically and numerically. In Fig. 9 the $x_{1}-t$,
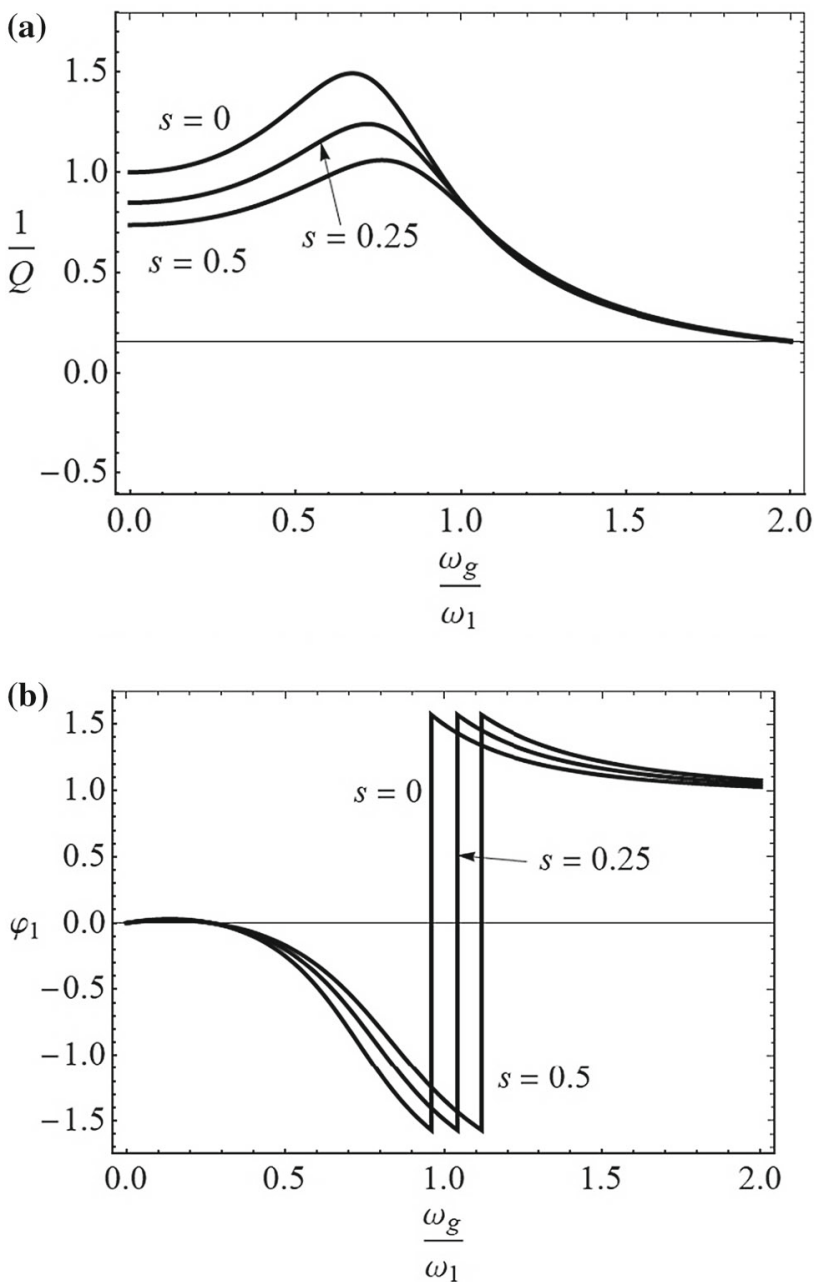

Fig. 8 a $1 / Q-\omega_{\mathrm{g}} / \omega_{1}$ diagrams for various $s ; \mathbf{b} \varphi_{1}-\omega_{\mathrm{g}} / \omega_{3}$ diagrams for various $s$

$x_{2}-t$ and $x_{3}-t$ diagrams for masses $m_{1}, m_{2}$ and $m_{3}$ are numerically obtained. Using the analytical procedure suggested in this paper and the relations (58), (61) and (62) the steady-state amplitudes $A_{1}, A_{2}$ and $A_{3}$ are computed. The calculated values are: $A_{1}=0.71439 \mathrm{~cm}$, $A_{2}=0.42857 \mathrm{~cm}$ and $A_{3}=0.14286 \mathrm{~cm}$.

Comparing the analytical and numerical results, it is seen that the difference is negligible. The analytical solutions are on the top of the numerical ones.

\section{Conclusion}

The approximate method based on rotating vectors for solving multi-degree-of-freedom nonlinear oscillatory system is developed. The presented methodology enable representation in a complex plane of rotating vectors which correspond to each mass of the system. The dynamic balance between internal and external forcing for each mass of the oscillator yields the fundamental resonance of the linear and damped system. The nonlinearity in the system is introduced as the cor- 

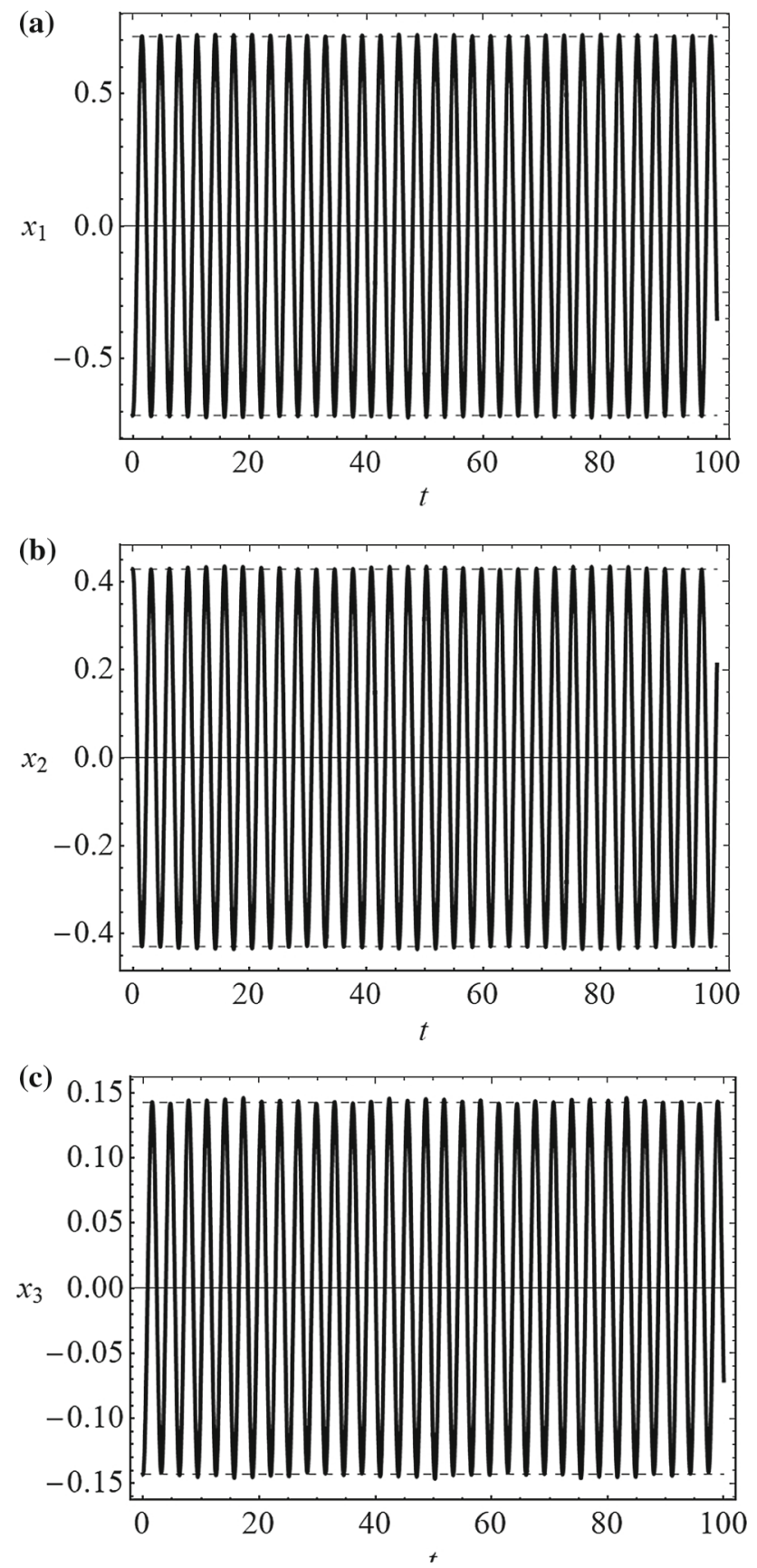

Fig. 9 a $x_{1}-t$ diagram (full line) and $A_{1}-t$ (dotted line) for $m_{1} ; \mathbf{b} x_{2}-t$ diagram (full line) and $A_{2}-t$ (dotted line) for $m_{2} ; \mathbf{c} x_{3}-t$ diagram (full line) and $A_{3}-t$ (dotted line) for $m_{3}$

rection to linear amplitudes of vibration. The suggested method is the generalization of the well-known procedure developed for a linear one degree-of-freedom oscillator. A difference in the multi degree of freedom system compared to one degree-of-freedom oscillator is that the force vector acts indirectly on all of masses in the system and the motion of each next mass in the system depends on the properties of the previous ones. The method is applied for a special case of a three mass system with three degrees of freedom The effect of nonlinearity on the vibration property of each of three mass is evident. It is concluded that the effect of nonlinearity on the amplitude of vibration decreases with higher values of the excitation frequency.

Funding Open access funding provided by Óbuda University.

Open Access This article is licensed under a Creative Commons Attribution 4.0 International License, which permits use, sharing, adaptation, distribution and reproduction in any medium or format, as long as you give appropriate credit to the original author(s) and the source, provide a link to the Creative Commons licence, and indicate if changes were made. The images or other third party material in this article are included in the article's Creative Commons licence, unless indicated otherwise in a credit line to the material. If material is not included in the article's Creative Commons licence and your intended use is not permitted by statutory regulation or exceeds the permitted use, you will need to obtain permission directly from the copyright holder. To view a copy of this licence, visit http://creativecomm ons.org/licenses/by/4.0/.

\section{References}

1. G. Genta, Vibration Dynamics and Control (Springer, Berlin, 2009)

2. M. Geradin, D.J. Rixen, Mechanical Vibrations: Theory and Application to Structural Dynamics (Wiley, Chichester, 2015)

3. A.F. Vakakis, A. Blanchard, J. Sound Vib. 413, 57 (2018)

4. R. Darula, S. Sorokin, J. Sound Vib. 385, 402 (2016)

5. Y.S. Hamed, M. Sayed, A.A. Alshehri, J. Vibroeng. 20(1), 42 (2018)

6. F. Robert, A. Agrawal, S. Clement, J. Electr. Electron. Eng. 13(1), 51 (2020)

7. J. Murin, J. Hrabovsky, J. Pantech, V. Kutis, F. Janicek, J. Electr. Eng. 63(7), 164 (2012)

8. P. Szuchy, I. Biro, in Proc. KOD 2018, Novi Sad, Serbia (2018)

9. L. Cveticanin, M. Zukovic, D. Cveticanin, J. Sound Vib. 436, 295 (2018)

10. L. Cveticanin, Strong Nonlinear Oscillator-Solutions, 2nd edn. (Springer, Berlin, 2018)

11. Y. Fu, J. Zhang, L. Wan, Appl. Phys. 11(3), 482 (2011)

12. H. Rafieipour, A. Lotfavar, A. Masroori, IJST 37(M2), $83(2013)$

13. M. Bayat, M. Bayat, I. Pakar, Lat. Am. J. Solids Struct. 11(3), 534 (2014)

14. S. Sadeghyadeh, A. Kaliri, Lat. Am. J. Solids Struct. 13, 478 (2016)

15. S. Peter, R.I. Leine, Mech. Syst. Signal Process. 96, 139 (2017)

16. R. Mickens, Mathematical Methods for the Natural and Engineering Sciences (World Scientific, New Jersey, 2004) 